\title{
The Swift-BAT survey reveals the orbital period of three high-mass X-ray binaries
}

\author{
A. D’Aì ${ }^{1}$, V. La Parola ${ }^{2}$, G. Cusumano ${ }^{2}$, A. Segreto ${ }^{2}$, P. Romano ${ }^{2}$, S. Vercellone ${ }^{2}$, and N. R. Robba ${ }^{1}$ \\ 1 Dipartimento di Scienze Fisiche ed Astronomiche, Università di Palermo, via Archirafi 36, 90123, Palermo, Italy \\ e-mail: dai@fisica.unipa.it \\ 2 INAF, Istituto di Astrofisica Spaziale e Fisica Cosmica, via U. La Malfa 153, 90146 Palermo, Italy \\ Received 23 December 2010 / Accepted 22 February 2011
}

\begin{abstract}
Aims. A growing number of previously hidden Galactic X-ray sources are now detected with recent surveys performed by the INTEGRAL and Swift satellites. Most of these new sources eluded past surveys due to their large local X-ray extinction and consequent low soft X-ray flux. The Swift-BAT performs daily monitoring of the sky in an energy band (15-150 keV) which is only marginally affected by X-ray extinction, thus allowing for the search of long periodicities in the light curve and identification of the nature of the X-ray sources.

Methods. We performed a period search using the folding technique in the Swift-BAT light curves of three INTEGRAL sources: IGR J05007-7047, IGR J13186-6257 and IGR J17354-3255. Their periodograms show significant peaks at $30.77 \pm 0.01 \mathrm{~d}$, $19.99 \pm 0.01 \mathrm{~d}$ and $8.448 \pm 0.002 \mathrm{~d}$, respectively. We estimate the significance of these features from the $\chi^{2}$ distribution of all the trials, finding a probability $\leq 1.5 \times 10^{-4}$ that the detections occurred due to chance. We complement our analysis with the study of their broadband X-ray emission.

Results. We identify the periodicities with the orbital periods of the sources. The periods are typical for the wind accretors X-ray binaries and we support this identification showing that also their energy spectra are compatible with an X-ray spectral emission characteristic of high-mass X-ray binaries. The spectrum of IGR J05007-704 that resides in the Large Magellanic Cloud, does not show any intrinsic local absorption, whereas the spectra of the Galactic sources IGR J17354-3255 and IGR J13186-6257 may be affected by a local absorber. The folded light curve for IGR J13186-6257 suggests a possible Be companion star.
\end{abstract}

Key words. X-rays: binaries - X-rays: individuals: IGR J05007-7047 - X-rays: individuals: IGR J13186-6257 X-rays: individuals: IGR J17354-3255

\section{Introduction}

One of the main results of the IBIS/ISGRI telescope (Ubertini et al. 2003) on board the INTEGRAL satellite (Winkler et al. 2003) is the detection of a large number of new hard $\mathrm{X}$-ray sources. At present, INTEGRAL has detected more than 500 sources $(\sim 26 \%$ are associated with extragalactic sources, $\sim 26 \%$ with Galactic sources and $\sim 48 \%$ whose nature has not yet been determined) ${ }^{1}$. Among the Galactic sources, 56 are identified as binary systems with a supergiant companion. Most of them, characterized by persistent emission, are likely deeply embedded in their stellar wind, as suggested by their strong intrinsic absorption $\left(N_{\mathrm{H}}>10^{23} \mathrm{~cm}^{2}\right.$, see e.g. Walter et al. 2006).

An important contribution to the study of these new Galactic sources is provided by the Burst Alert Telescope (BAT, Barthelmy et al. 2005) on board Swift (Gehrels et al. 2004), which has been performing a continuous monitoring of the sky in the hard X-ray energy range (15-150 keV) since November 2004. The telescope, thanks to its large field of view (1.4 steradians half coded) and its pointing strategy, covers a fraction between $50 \%$ and $80 \%$ of the sky every day. This has allowed the detection of many of the new INTEGRAL HMXBs (e.g. Cusumano et al. 2010b) and the collection of their long term

\footnotetext{
1 An updated list of these sources with their main properties and the relevant references can be found in http://irfu.cea.fr/Sap/ IGR-Sources/
}

light curves and of their averaged X-ray spectra. The long and continuous monitoring of these sources allows to investigate the intrinsic emission variability, to search for long periodicities (orbital periods) and to discover the presence of eclipse events. The role of Swift-BAT is therefore fundamental to unveil the nature and the geometry of these X-ray binary systems.

We are performing a systematic study of the light curves of the new INTEGRAL sgHMXB sources to search for $\geq 0.5$ day periodicities and to discover the presence of eclipse events allowing the study of the binary system geometry. In this paper we present the detailed timing analysis and broadband $(0.2-150 \mathrm{keV})$ spectral analysis of IGR J05007-7047, IGR J13186-6257, and IGR J17354-3255.

IGR J05007-7047 (also known as IGR J05009-7044), located in the Large Magellanic Cloud (LMC), was detected with INTEGRAL in the $17-60 \mathrm{keV}$ band (Sazonov et al. 2005) with a flux of $1.2 \times 10^{-11} \mathrm{erg} \mathrm{cm}^{-2} \mathrm{~s}^{-1}$, corresponding to a luminosity of $3.6 \times 10^{36} \mathrm{erg} \mathrm{s}^{-1}$, assuming a distance of $50 \mathrm{kpc}$ (Guinan et al. 1998). A follow-up Chandra observation allowed its association with the relatively bright blue $(V=14.8 \mathrm{mag}$, $B-V=-0.01 \mathrm{mag}$ ) star USNO-B1.0 0192-0057570 (Halpern 2005) of spectral type B2 III (Masetti et al. 2006) at a redshift consistent with that of LMC. La Parola et al. (2010b) reported on the detection in the BAT survey data of a periodic modulation at $30.77 \mathrm{~d}$, later confirmed by Coe et al. (2010) through the analysis of the optical counterpart light curve. 
Table 1. Log of Swift-BAT and Swift-XRT observations.

\begin{tabular}{lccccc}
\hline \hline Source & $\begin{array}{c}\Delta T_{\text {BAT }} \\
\mathrm{d}\end{array}$ & $\begin{array}{c}\text { BAT exposure } \\
\mathrm{d}\end{array}$ & XRT ObsId & $\begin{array}{c}\text { Obs. date } \\
\text { yyyy-mm-dd }\end{array}$ & $\begin{array}{c}\text { XRT exposure } \\
\mathrm{s}\end{array}$ \\
\hline IGR J05007-7047 & 1644.44 & 178.27 & 00031846001 & $2010-10-25$ & 1431 \\
& & & 00031846002 & $2010-10-27$ & 971 \\
& & & 00031846003 & $2010-10-28$ & 6197 \\
IGR J13186-6257 & 1640.80 & 119.73 & 00037071001 & $2008-05-01$ & 740 \\
& & & 00037071002 & $2010-05-25$ & 1532 \\
IGR J17354-3255 & 1609.18 & \multirow{2}{*}{130.30} & 00037054001 & $2008-03-11$ & 4408 \\
& & & 00037054002 & $2009-04-17$ & 5285 \\
\hline
\end{tabular}

IGR J13186-6257 was first reported in the Third Integral Catalogue (Bird et al. 2007) as an unidentified hard X-ray source. A follow-up Swift-XRT observation better constrained its position and revealed a flat soft X-ray spectrum (Landi et al. 2008). A short ( $\sim 5 \mathrm{ks})$ Chandra observation found an X-ray source within the Swift-XRT error box at (RA, Dec $[\mathrm{J} 2000]=199.604500 \mathrm{deg},-62.970972 \mathrm{deg}$ ) (Tomsick et al. 2009). The Chandra source, CXOU J131825.0-625815, was identified as the most likely counterpart of the INTEGRAL source, with a low spurious detection probability of $\sim 5 \%$. The Chandra position allowed for the counterpart optical identification with the 2MASS source J13182505-6258156. The spectral shape of the source was consistent with a highly absorbed $\left(1.8 \times 10^{23} \mathrm{~cm}^{-2}\right)$ power-law spectrum.

IGR J17354-3255 was discovered during the INTEGRAL Galactic Bulge monitoring program (Kuulkers et al. 2006, 2007). The source has also a possible, highly variable, $\gamma$-ray counterpart detected with the AGILE satellite (AGL J1734-3310, Bulgarelli et al. 2009). A Swift-XRT follow-up observation (Vercellone et al. 2009) revealed the presence of two sources within the INTEGRAL error circle. Based on its variability, the authors suggested the source at $(\mathrm{RA}$, Dec $[\mathrm{J} 2000]=263.863167 \mathrm{deg}$, $-32.930250 \mathrm{deg}$ ) as the likely counterpart of IGR J17354-3255. The spectrum of this source is characterized by strong absorption $\left(N_{\mathrm{H}}=5 \pm 2 \times 10^{22} \mathrm{~cm}^{-2}\right)$ and high flux variability (an order of magnitude change in flux within $7 \mathrm{~h}$ ). A further Chandra observation (Tomsick et al. 2009) confirmed its variable nature and suggested the association with the IR source 2MASS J17352760-3255544. A flux modulation in the SwiftBAT data, with a period of 8.452 days, has been first reported by D’Aì et al. (2010).

This paper is organized as follows. Section 2 describes the data reduction. In Sects. 3 and 4 we describe the temporal and spectral analysis and in Sect. 5 we discuss our results.

\section{Data reduction}

The raw BAT survey data of the first 54 months of the Swift mission were retrieved from the HEASARC public archive ${ }^{2}$ and processed with a dedicated software (Segreto et al. 2010), that performs screening, mosaicking and source detection on Swift-BAT data and produces spectra and light curves for any given sky position. IGR J05007-7047, IGR J13186-6257 and IGR J17354-3255 were detected in the Swift-BAT all-sky map with a significance maximized in the $15-50 \mathrm{keV}$ band of 16.1 , 10.0, and 18.9 standard deviations, respectively. Light curves were extracted in the $15-150 \mathrm{keV}$ energy range with the maximum available time resolution ( $\sim 300 \mathrm{~s})$ and corrected to the solar system barycentre (SSB) by using the task EARTH2sun. The 15-150 keV spectra were obtained extracting the source fluxes

\footnotetext{
$\overline{2}$ http://heasarc.gsfc.nasa.gov/docs/archive.html
}

from the sky maps in sixteen energy bands, and were analyzed using an appropriately rebinned CALDB response matrix.

Swift-XRT observed IGR J05007-7047 between 2010-1025 and 2010-10-28 (ObsId 31846); IGR J13186-6257 was observed on 2008-05-01 and on 2010-05-25 (ObsId 37071); IGR J17354-3255 was observed on 2008-03-11 and 2009-0417 (ObsId 37054). The sources were all observed in Photon Counting mode. Source and background spectra and the relevant ancillary files were created and retrieved from the Swift-XRT product on-line builder maintained by the Swift data center at the University of Leicester (Evans et al. 2009). The Swift-XRT spectra were rebinned to have at least 20 counts per bin, and Swift-BAT energy channels were grouped to obtain a $S / N$ ratio $\geq 3$ per channel. This choice allows us the use of the $\chi^{2}$ statistics. In the following, we report errors at $90 \%$ confidence level, if not stated otherwise.

Table 1 reports the log of the Swift-BAT and Swift-XRT observations used for the present analysis.

\section{Temporal analysis}

We analyzed the long term Swift-BAT light curve to search for intensity modulations by applying a folding technique and searching in the $0.5-100 \mathrm{~d}$ period range. The period resolution is given by $P^{2} /\left(N \Delta T_{\mathrm{BAT}}\right)$, where $N=16$ is the number of trial profile phase bins and $\Delta T_{\mathrm{BAT}}$ is the data span length (Buccheri \& Sacco 1985).

The average rate in each phase bin was evaluated by weighting the light curve rates by the inverse square of the corresponding statistical error

$R_{j}=\frac{\sum r_{i} / e r_{i}^{2}}{\sum 1 / e r_{i}^{2}}$

where $R_{j}$ is the average rate in the $j$ th phase bin of the trial profile, $r_{i}$ are the rate of the light curve whose phase fall into the $j$ th phase bin and $e r_{i}$ are the corresponding statistical errors. The error on $R_{j}$ is $\left(\sqrt{\sum 1 / e r_{i}^{2}}\right)^{-1}$. The weighting procedure was adopted to deal with the large span of $e r_{i}$ and it is justified because the BAT data are background dominated.

The significance of a feature $P_{\mathrm{o}}$ in the resulting periodogram cannot be evaluated using the $\chi^{2}$ statistics because, if the source is variable on time scales comparable to those under investigation, the average $\chi^{2}$ is far from the value expected for white noise $(N-1)$. Therefore, the significance of $P_{\mathrm{o}}$ is evaluated as follows (La Parola et al. 2010a; Cusumano et al. 2010a):

1. we fit each periodogram with a second order polynomial and subtract the trend to the $\chi^{2}$ distribution, obtaining a new $\chi_{\mathrm{C}}^{2}$ distribution; 
2. we build the histogram of the new $\chi_{\mathrm{C}}^{2}$ distribution excluding the interval around $P_{\mathrm{o}}$ and those around any features deriving from it (e.g. periods multiple of $P_{\mathrm{o}}$ );

3 . we fit the tail of the resulting distribution with an exponential function and evaluate the integral of the best-fit function beyond $\chi_{\mathrm{C}}^{2}\left(\mathrm{P}_{\mathrm{o}}\right)$. This integral yields the number of chance occurrences due to noise.

\subsection{IGR J05007-7047}

Figure 1a shows the periodogram obtained for IGR J05007-7047. We find significant evidence for the presence of a periodicity $\left(\chi^{2} \sim 210\right)$ at a period of $P_{0}=30.77 \pm 0.03 \mathrm{~d}$, where the period uncertainty is the period resolution at $P_{\mathrm{o}}$. The other two significant features in the periodogram correspond to $2 P_{\mathrm{o}}$ and $3 P_{\mathrm{o}}$. The value of $\chi_{\mathrm{C}}^{2}$ (i.e. $\chi^{2}$ after subtracting the periodogram trend) at $P_{\mathrm{o}}$ is 177 . Figure $1 \mathrm{~b}$ shows the $\chi_{\mathrm{C}}^{2}$ distribution excluding the values around $P_{\mathrm{o}}$ and its multiples. We then fitted the tail $\left(\chi_{\mathrm{C}}^{2}>15\right)$ of the resulting distribution with an exponential function and evaluated the integral of the best-fit function beyond $P_{0}$. The number of chance occurrences to have a value of 177 or higher is $3.9 \times 10^{-12}$, that corresponds to $\sim 7$ standard deviations in Gaussian statistics. The pulsed profile (Fig. 1c) folded at $P_{\mathrm{o}}$ with $T_{\text {epoch }}=54159.753 \mathrm{MJD}$, shows a roughly sinusoidal modulation with a minimum value consistent with zero intensity. The centroid of the minimum, evaluated by fitting the data around the dip with a Gaussian model is at phase 0.23 . The uncertainty depends on the rough binning of the folded light curve and we conservatively assume an error corresponding to one bin phase; the dip phase, therefore, corresponds to MJD (54 166.8 \pm 1.0$) \pm n \times P_{\mathrm{o}}$ MJD. The phase coverage of the light curve bins shows that this dip is not due to an accidental under-sampling of the light curve at this phase. We self-consistently checked this aspect also for the dips of IGR J17354-3255 and IGR J13186-6257.

The three Swift-XRT observations were performed at the orbital phase intervals of $0.369-0.380,0.428-0.432$ and $0.469-0.482$, respectively. The $0.2-10 \mathrm{keV}$ light curve shows a persistent emission with a variability of a factor $\sim 5$ and an average count rate of $0.058 \pm 0.004$ count s$^{-1}$. The source events selected in the three observations are 77, 37 and 509, respectively. Because of the low statistic content of the first 2 observations we restricted the analysis to ObsId 00031846003.

\subsection{IGRJ13186-6257}

The highest feature, with a $\chi^{2}$ value of $\sim 164$, is at $P_{\mathrm{o}}=19.99 \pm$ $0.01 \mathrm{~d}$ (Fig. 2a). The second and third highest peaks in this periodogram are found at multiple values of $P_{\mathrm{o}}$ at $\sim 40 \mathrm{~d}$ and $60 \mathrm{~d}$. We fit the periodogram with a second order polynomial and subtracted the trend from the $\chi^{2}$ distribution, finding a $\chi_{\mathrm{C}}^{2}=126$ at $P_{\mathrm{o}}$. We therefore built the histogram of the $\chi_{\mathrm{C}}^{2}$ distribution (Fig. 2b) excluding the interval around $P_{\mathrm{o}}$ and the first two multiples of $P_{\mathrm{o}}$. The integral above $P_{\mathrm{o}}$ yields a number of chance occurrences due to noise of $3.2 \times 10^{-6}$, corresponding to a significance for the detected feature of $\sim 4.7$ standard deviations in Gaussian statistics.

The pulsed profile (Fig. 2c) folded at $P_{\mathrm{o}}$ with $T_{\text {epoch }}=$ 54 417.993 MJD, shows a plateau between phase 0.9 and 1.4, where source emission is dominated by background, whereas the source becomes clearly detected in the remaining half of the orbit. We evaluated the time passage corresponding to the folded light curve minimum bin at phase 0.95 corresponding to
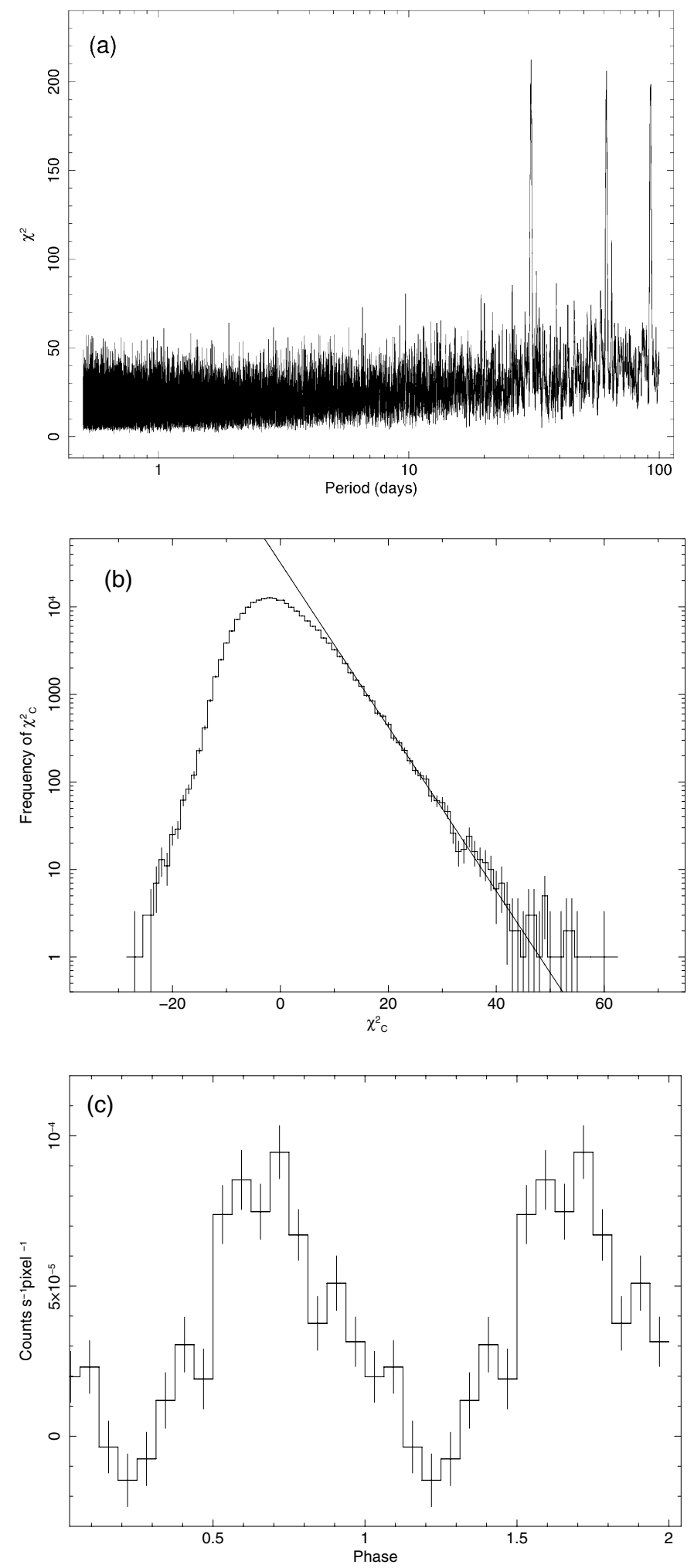

Fig. 1. a) Periodogram of Swift-BAT $(15-50 \mathrm{keV})$ data for IGR J05007-7047. b) Distribution of $\chi_{\mathrm{C}}^{2}$ values excluding the intervals around $P_{\mathrm{o}}$ and its multiples. The continuous line is the best fit obtained with an exponential model applied to the tail of the distribution $\left(\chi^{2}>15\right)$. c) Swift-BAT light curve folded at $P=30.77 \pm 0.01 \mathrm{~d}$ period in 16 phase bins.

$T_{\text {dip }}=(54436.2 \pm 0.6) \pm n \times P_{\mathrm{o}}$ MJD. The two Swift-XRT observations were performed at orbital phases 0.50 and at phase 0.20 . The source is clearly detected in the first observation with an 

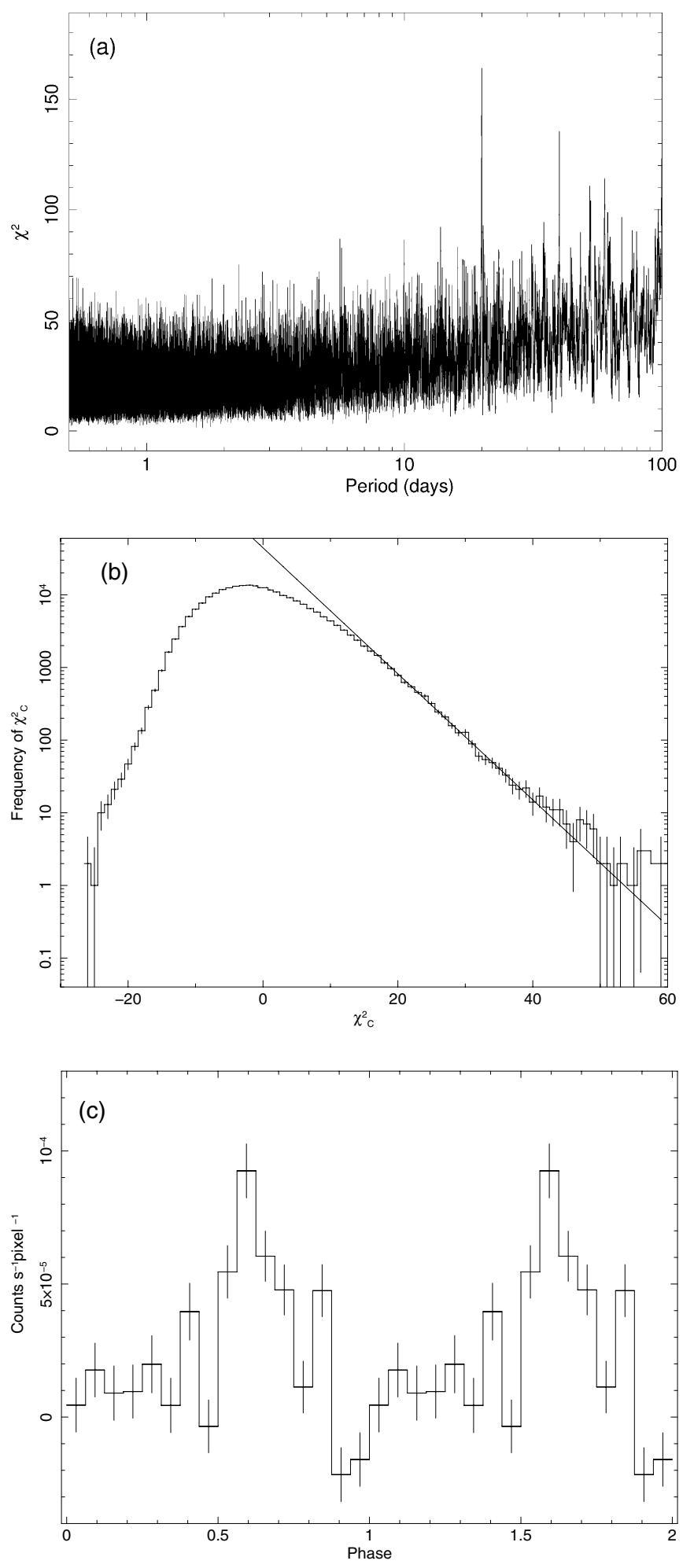

Fig. 2. a) Periodogram of Swift-BAT $(15-50 \mathrm{keV})$ data for IGR J13186-6257. b) Distribution of $\chi_{\mathrm{C}}^{2}$ values excluding the intervals around $P_{\mathrm{o}}$ and its multiples. The continuous line is the best fit obtained with an exponential model applied to the tail of the distribution $\left(\chi^{2}>15\right)$. c) Swift-BAT light curve folded at $P=19.99 \pm 0.01 \mathrm{~d}$ period in 16 phase bins.

averaged count rate of $0.28 \pm 0.02$ count $^{-1}$. In the second observation, performed two years later, no source is detected at the same celestial coordinates. We estimated an upper limit on the source flux between 1.2 and $1.5 \times 10^{-12} \mathrm{erg} \mathrm{cm}^{-2} \mathrm{~s}^{-1}$ depending on the choice of the continuum as derived from the first observation. For spectral analysis we made use only of the data from the first observation.

\subsection{IGR J17354-3255}

The periodogram obtained for IGR J17354-3255 (Fig. 3a) shows significant evidence for the presence of a periodicity $\left(\chi^{2} \sim 121\right)$ at a period of $P_{\mathrm{o}}=8.448 \pm 0.002 \mathrm{~d}$. The other significant features that appear in the periodogram corresponds to multiple of $P_{\mathrm{o}}$ (up to $8 P_{\mathrm{o}}$ ). After correcting for the periodogram trend, we obtain $\chi_{\mathrm{C}}^{2}\left(P_{\mathrm{o}}\right)=94$. Figure $3 \mathrm{~b}$ shows the $\chi_{\mathrm{C}}^{2}$ distribution excluding the values around $P_{\mathrm{o}}$ and its multiples. The number of chance occurrences to have a value of 94 or higher is $1.5 \times 10^{-4}$ that corresponds to $\sim 4$ standard deviations in Gaussian statistics. The pulsed profile (Fig. 3c) folded at $P_{\mathrm{o}}$ with $T_{\text {epoch }}=54175.159 \mathrm{MJD}$, shows a modulation with a minimum value consistent with zero intensity. The centroid of the minimum is at phase 0.78 corresponding to MJD $(54181.75 \pm 0.26) \pm n \times P_{\mathrm{o}}$ MJD.

The two Swift-XRT observations were performed at the orbital phase intervals of $0.719-0.767$ and $0.304-0.338$, respectively. The phase of first observation corresponds to the minimum in the folded profile (Fig. 3c), while the phase of the second one falls around the maximum of the profile. Figure 4 shows the Swift-XRT field of view of the two observations: the position of the two possible counterparts reported in Vercellone et al. (2009) are marked as Src1 (RA, Dec [J2000] = 263.863167 deg, $-32.930250 \mathrm{deg})$ and Src2 (RA, Dec [J2000] = $263.826333 \mathrm{deg}$, $-32.906361 \mathrm{deg})$. Src1 is not detected in the first observation, with a $3 \sigma$ upper limit of $2.3 \times 10^{-3}$ count $^{-1}$. As this observation corresponds to a phase consistent with the minimum of the BAT folded light curve we can confidently associate Src1 to IGR J17354-3255. In the second observation the $0.2-10 \mathrm{keV}$ average count rate of $\mathrm{Src} 1$ is $0.076 \pm 0.004$ count s$^{-1}$, with a variability of a factor $\sim 20$.

\section{Spectral analysis}

We have performed a broad band spectral analysis of the XRT and BAT data. The XRT and BAT spectra are not simultaneous, as the BAT spectra are accumulated over 54 months. Therefore, we have checked for the presence of spectral variability in the BAT data by building the hardness ratio defined as the ratio between the count rate in the $15-150 \mathrm{keV}$ and in the $15-35 \mathrm{keV}$ energy band, with a time resolution of 1 day. We found no significant variation of the hardness ratio along time for the three sources. We also verified that the BAT count rate during the XRT observations is consistent with the average count rate in the 54 months. However, to take into account residual flux and intercalibration uncertainties between the two spectra, we included a multiplicative constant factor $\left(C_{\mathrm{BAT}}\right)$ in all the models. We kept it frozen to unity for XRT data and free to vary for BAT data. Because of possible correlations between the values of $N_{\mathrm{H}}$ and the indeces of the power-laws, we built 2D contour-plots around the best-fitting values, always checking the consistency of the error estimates. We report in Table 2 the spectral best-fitting results for the three sources, using a cut-off power-law model. SwiftBAT fluxes include the $C_{\mathrm{BAT}}$ factor in the calculation. 

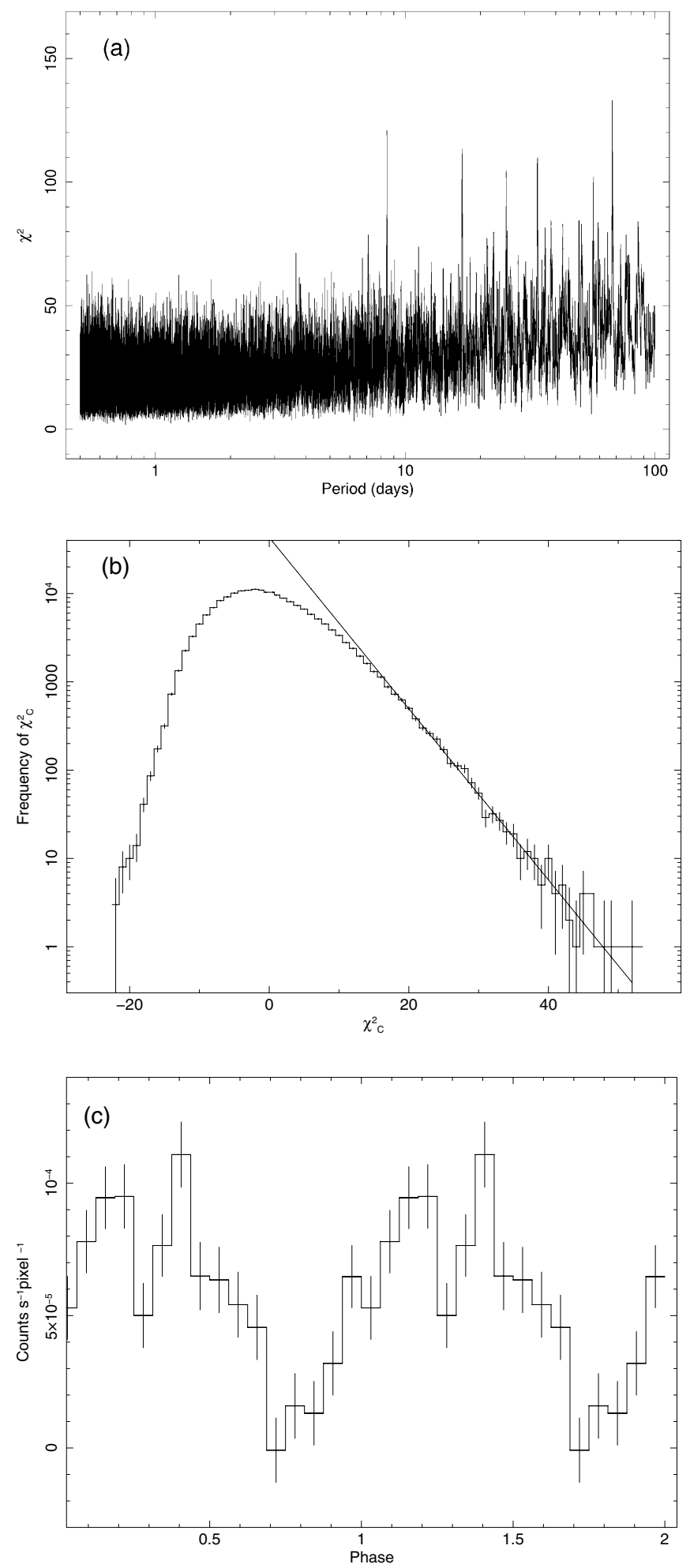

Fig.3. a) Periodogram of Swift-BAT $(15-50 \mathrm{keV})$ data for IGRJ17354-3255. b) distribution of $\chi_{\mathrm{C}}^{2}$ values excluding the intervals around $P_{\mathrm{o}}$ and its multiples. The continuous line is the best fit obtained with an exponential model applied to the tail of the distribution $\left(\chi^{2}>15\right)$. c) Swift-BAT Light curve folded at $P=8.452 \pm 0.002 \mathrm{~d}$ period in 16 phase bins.

\subsection{IGR J05007-7047}

The combined XRT-BAT spectrum was first modelled with a simple absorbed power law. The resulting $\chi^{2}$ (140.2 with 38 degrees of freedom, d.o.f.) was not acceptable, and the residuals suggested the presence of a cut-off at high energy. We have then included such cut-off (model cuTOFFPL), obtaining an improved $\chi^{2}$ of 32.0 for 37 d.o.f., with a cut-off at $E_{\mathrm{C}}=12 \pm 2 \mathrm{keV}$ and a photon-index $\Gamma=0.3 \pm 0.2$. The interstellar absorption is consistent with the expected value in the direction of LMC (Kalberla et al. 2005). Because of the unambiguous identification of the optical counterpart as belonging to the LMC, we derive an extrapolated $0.2-150 \mathrm{keV}$ X-ray luminosity of $\sim 9 \times 10^{36} \mathrm{erg} \mathrm{s}^{-1}$. Figure 5a shows the spectrum, best fit model and residuals in units of standard deviations.

\subsection{IGRJ13186-6257}

An absorbed power-law model can satisfactorily model the data $\left(\chi^{2} /\right.$ d.o.f. $\left.=24 / 21\right)$. For this model the $N_{\mathrm{H}}$ is $17_{-5}^{+6} \times 10^{22} \mathrm{~cm}^{-2}$, the power-law photon index is $2.3 \pm 0.3$. The costant of normalization between the Swift-XRT and Swift-BAT data is, however, inconsistent with unity (being $0.2_{-0.08}^{+0.14}$ ). We found only marginal evidence for a high-energy cut-off in the spectrum $\left(E_{\text {cut }}>10 \mathrm{keV}\right)$, with a new $\chi^{2} /$ d.o.f. of $21 / 20$. The position of the high-energy cut-off strongly depends on the value of the $N_{\mathrm{H}}$ and of the photon-index. Choosing a reference value for $E_{\text {cut }}$ at $16 \mathrm{keV}$, we found an upper limit to the $N_{\mathrm{H}}$ at $11 \times 10^{22} \mathrm{~cm}^{-2}$ and a photonindex constrained in the $0-1.0$ range. Using this model the $C_{\mathrm{BAT}}$ value is very low (see Table 2 ) and this suggests strong variability, probably related to the orbital phase. Taking into account the time of the Swift-XRT observation, occured at phase 0.5 , we extracted a phase-selected Swift-BAT spectrum, choosing the interval $0.5-0.8$ as representative of the phase where source emission is most clearly detected above background. In this case, the $C_{\mathrm{BAT}}$ parameter assumes the value $0.28_{-0.12}^{+0.22}$, while the spectral parameters are still consistent with those obtained using the phase-averaged spectrum. The sensible change in the $C_{\mathrm{BAT}}$ parameter value favors a scenario where the X-ray emission may be confined only in a restricted orbital phase interval. We show in Fig. 5b, the best-fitting model together with data (using the Swift-BAT phase-averaged spectrum) and residuals in units of $\sigma$.

\subsection{IGR J17354-3255}

The fit of the combined XRT-BAT spectrum with a simple absorbed power-law model gives a statistically acceptable result $\left(\chi^{2} /\right.$ d.o.f. $\left.=29.6 / 27\right)$, with a $N_{\mathrm{H}}$ value of $10.6_{-1.4}^{+1.7} \times 10^{22} \mathrm{~cm}^{-2}$ and a photon-index of $2.6 \pm 0.3$. However, fitting the data with a cutoff power law, we obtained a significant improvement of the fit, with a final $\chi^{2} /$ d.o.f. of $23.2 / 26$, and an F-test probability of $\sim 1 \%$ of obtaining this improvement by chance. The cut-off energy is however poorly constrained and we obtained only a lower limit at $\sim 18 \mathrm{keV}$. The photon index $\Gamma$ is between 1.0 and 2.0, while the $N_{\mathrm{H}}$ is $7 \pm 2 \times 10^{22} \mathrm{~cm}^{-2}$. The expected $N_{\mathrm{H}}$ value in the direction of the source is considerably less $\left(1.2-1.6 \times 10^{22} \mathrm{~cm}^{-2}\right)$, and this suggests that a fraction of the X-ray absorbing medium could be local to the X-ray source.

The source position is only at $5^{\circ}$ from the Galactic Center (GC), and if we assume the distance of the GC as the possible distance of the source $(8 \mathrm{kpc})$, we obtain an extrapolated X-ray luminosity of $\sim 5 \times 10^{35} \mathrm{erg} \mathrm{s}^{-1}$. We show in Fig. $5 \mathrm{c}$, the bestfitting model together with data and residuals in units of $\sigma$.

\section{Discussion and conclusions}

In the last census of high-mass X-ray binaries in the Galaxy (Liu et al. 2006), there were 114 HMXBs sources reported, with only 
Table 2. Spectral fitting results.

\begin{tabular}{llll}
\hline \hline & IGR J05007-7047 & IGR J13186-6257 & IGR J17354-3255 \\
\hline$N_{\mathrm{H}}\left(10^{22} \mathrm{~cm}^{-2}\right)$ & $<0.17$ & $6_{-4.5}^{+5}$ & $7 \pm 2$ \\
$\Gamma$ & $0.3 \pm 0.2$ & $0.5 \pm 0.5$ & $1.7_{-0.7}^{+0.6}$ \\
$E_{\text {cut }}(\mathrm{keV})$ & $12 \pm 2$ & $16^{a}$ & $>18$ \\
Flux $^{b} 0.2-10 \mathrm{keV}$ & $0.86_{-0.16}^{+0.13}$ & $5.7_{-3.0}^{+0.6}$ & $1.1_{-0.4}^{+0.1}$ \\
Flux $^{b} 10-100 \mathrm{keV}$ & $1.9_{-0.6}^{+0.3}$ & $1.6 \pm 0.4$ & $2.6_{-1.4}^{+0.2}$ \\
$\chi^{2} /$ d.o.f. & $25.4 / 32$ & $21.5 / 20$ & $23.2 / 26$ \\
$C_{\text {BAT }}$ & $0.8_{-0.2}^{+0.4}$ & $0.06_{-0.02}^{+0.14}$ & $1.4_{-0.7}^{+1.1}$ \\
\hline
\end{tabular}

Notes. Summary of spectral fits using the cuToFfPL model for the combined Swift-XRT and Swift-BAT datasets. ${ }^{(a)}$ Parameter frozen in the fitting. (b) Flux in units of $10^{-11} \mathrm{erg} \mathrm{cm}^{-2} \mathrm{~s}^{-1}$.
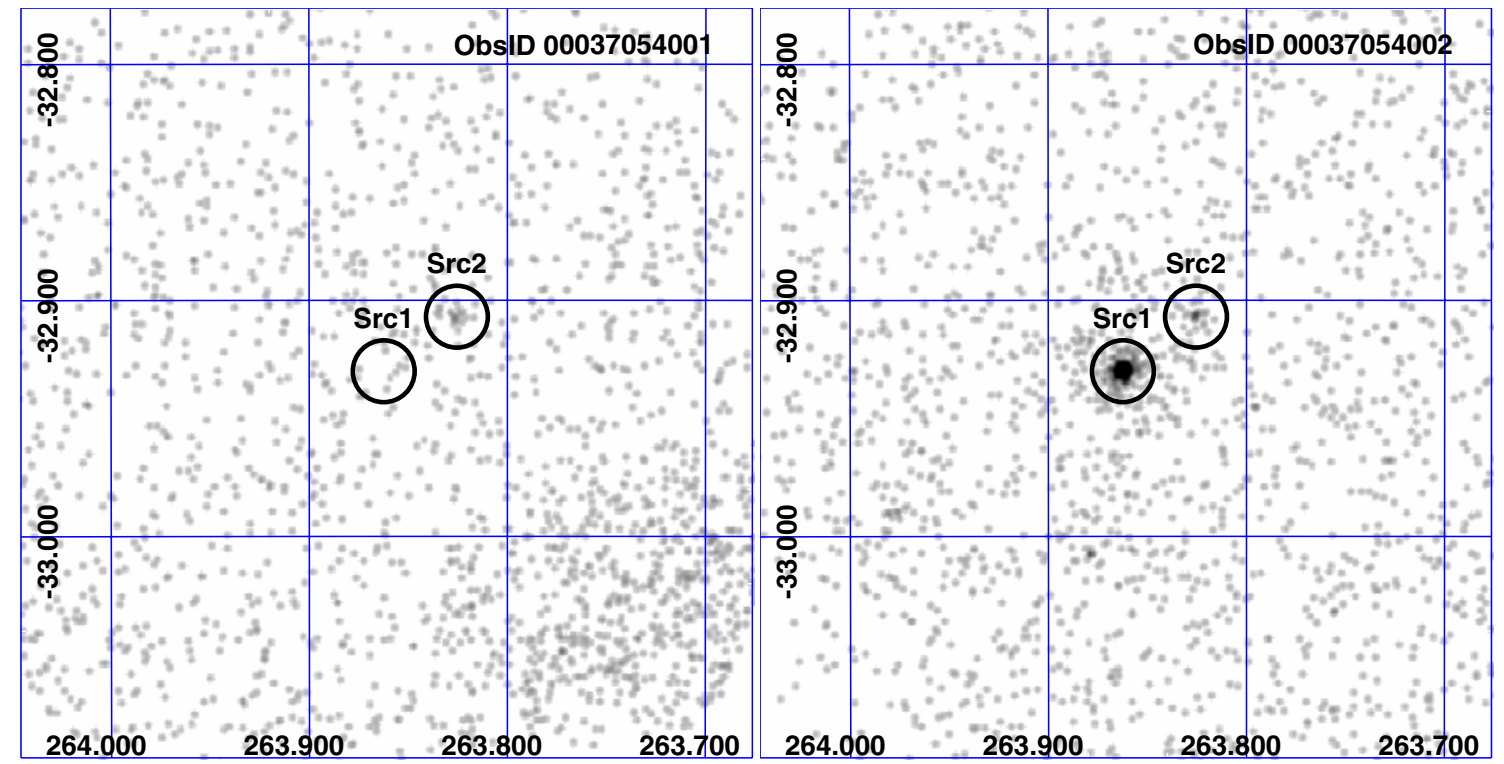

Fig. 4. Field of view of the two XRT observations of IGR J17354-3255, with the position of the two candidate counterparts (Vercellone et al. 2009).

50 determined orbital periods. With an increasing number of new detected X-ray sources, our view of HMXBs is rapidly changing (see Chaty et al. 2010), also due to the ability of the long term operating surveys to search for periodic signals.

We are currently undergoing a vast program for the search of periodic signals exploiting the Swift-BAT surveys (La Parola et al. 2010a; Cusumano et al. 2010a).

In this work, we have shown results from the analysis of the data collected by Swift-BAT during the first 54 months of the Swift mission for three still poorly understood INTEGRAL X-ray sources: IGR J05007-7047, IGR J13186-6257 and IGR J17354-3255.

IGR J05007-7047 was already identified as a possible windfed X-ray system, thanks to the association of the optical counterpart (Halpern 2005). We detect it in the BAT survey at a significance level of $\sim 16$ standard deviations with an average flux of $\sim 1.3 \times 10^{-11} \mathrm{erg} \mathrm{cm}^{-2} \mathrm{~s}^{-1}$ in the $15-50 \mathrm{keV}$ energy band. The light curve reveals a periodicity of $30.77 \pm 0.01 \mathrm{~d}$ that we interpret as the orbital period of the binary system. Given the characteristics of the companion star (spectral type B2 III, Masetti et al. 2006) we can apply Kepler's third law to derive the semi-major axis of the binary system. This is given by $a^{3}=P_{\text {orb }}^{2} \times G\left(M_{\star}+M_{\mathrm{X}}\right) / 4 \pi^{2}$, where $M_{\star}$ and $M_{\mathrm{X}}$ are the masses of the supergiant and compact object, respectively. We adopt $M_{\mathrm{X}}=1.4 M_{\odot}$ and $7<M_{\star}<12 M_{\odot}$ (Habets \& Heintze 1981 , for a B2 star of radius $4 R_{\odot}<R_{\star}<5.6 R_{\odot}$ ). This yields
$84 R_{\odot}<a<98 R_{\odot}\left(17 R_{\star}<a<21 R_{\star}\right)$. The folded profile shows a large dip at time of $(54166.8 \pm 0.6) \pm n P_{\mathrm{o}}$ MJD with a count rate consistent with no emission. However, the width of this dip ( $\sim 20$ per cent of $\left.P_{\mathrm{o}}\right)$ is not well constrained, constituting a rough upper limit too large to be consistent with the duration of the eclipse expected for a circular orbit with the inferred semimajor axis.

The spectrum of IGR J05007-7047 is well modelled with cut-off power law $\left(\Gamma \sim 0.3\right.$ and $\left.E_{\mathrm{c}} \sim 12 \mathrm{keV}\right)$. The upper limit on the absorbing column is consistent with the Galactic value along the line of sight. Therefore this source cannot be classified as an absorbed high-mass X-ray binary $\left(\geq 10^{23} \mathrm{~cm}^{-2}\right)$.

The spectral shape of IGR J13186-6257 could not be firmly constrained, because of the weak statistics of the Swift-XRT spectrum. We could not determine any clear cut-off in the energy spectrum, with a lower limit at $\sim 10 \mathrm{keV}$. The periodicity of $19.99 \mathrm{~d}$, is typical for wind/Be accreting X-ray systems. The folded light curve shows clear source emission above background only for $\sim$ half of the orbital period. Because of the poor constraints on the spectral shape, we are not able to univocally classify the source. However, the shape of the folded light curve, the very low value of the intercalibration constant between the pointed Swift-XRT spectrum and the long-term Swift-BAT spectrum and the lack of source detection at orbital phase 0.2 suggest a possible Be companion in an eccentric orbit, where accretion could take place only near the peri-astron passage. The expected 

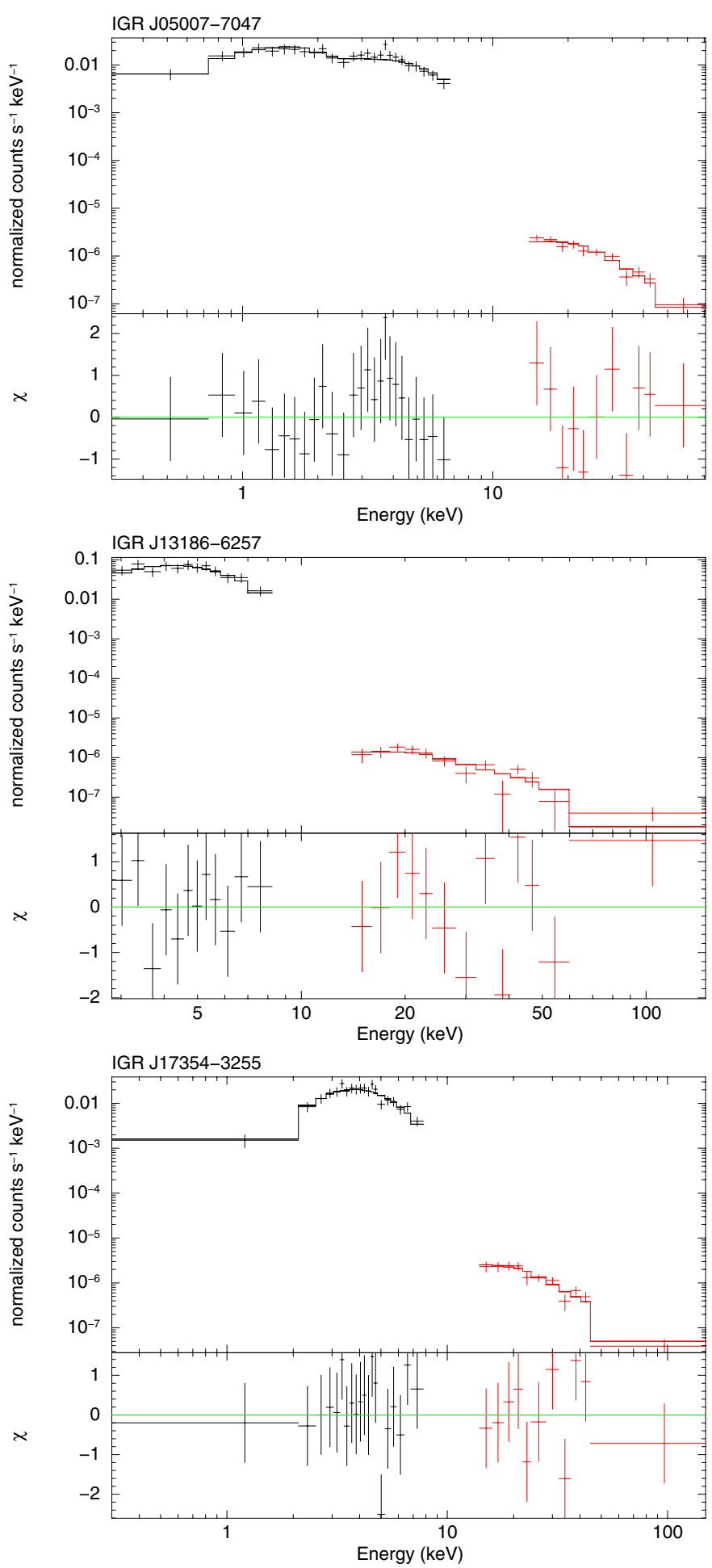

Fig. 5. Combined Swift-XRT and Swift-BAT spectra, best-fitting models (see Table 2) and residuals in units of standard deviations of IGR J05007-7047 (upper panel), IGR J13186-6257 (middle panel) and IGR J17354-3255 (bottom panel).

equivalent column density value in the IGR J13186-6257 direction lies between $1.24 \times 10^{22} \mathrm{~cm}^{-2}$ (Kalberla et al. 2005) and $1.59 \times 10^{22} \mathrm{~cm}^{-2}$ (Dickey \& Lockman 1990). These estimates are considerably less than our best-fitting values, both the simple power-law and for the cut-off power-law model.
IGR J13186-6257 may be therefore embedded in a denser absorbing medium during accretion phases. Further observations are, however, necessary to confirm this scenario.

IGR J17354-3255 is detected in the $15-50 \mathrm{keV}$ Swift-BAT map at a significance level of $\sim 19$ standard deviations. The average flux in this band is $\sim 1.56 \times 10^{-11} \mathrm{erg} \mathrm{cm}^{-2} \mathrm{~s}^{-1}$. The timing analysis on the 15-50 Swift-BAT light curve unveils an orbital period of $8.448 \pm 0.002 \mathrm{~d}$, with a significance of $\sim 4$ standard deviations, confirming its nature as a binary system. The detection of the periodicity in enforced by the non-detection of the soft X-ray counterpart in the Swift-XRT observation performed at an orbital phase consistent with the minimum of the modulation. The folded profile shows a minimum consistent with zero intensity. The energy spectrum of IGR J17354-3255 is well modelled by an absorbed power law, with $N_{\mathrm{H}} \sim 7 \times 10^{22} \mathrm{~cm}^{-2}$ with a cutoff at $>20 \mathrm{keV}$. This large local absorption suggests that X-ray emission from the compact object may interact with the stellar wind of the companion star, whose spectral type has not yet been determined.

Acknowledgements. This work made use of data supplied by the UK Swift Science Data Centre at the University of Leicester. A.D'Aì acknowledges financial contribution from the agreement ASI-INAF I/009/10/0. The authors wish to thank the anonymous referee for comments that helped us to improve the paper.

\section{References}

Barthelmy, S. D., Barbier, L. M., Cummings, J. R., et al. 2005, Space Sci. Rev., 120,143

Bird, A. J., Malizia, A., Bazzano, A., et al. 2007, ApJS, 170, 175

Buccheri, R., \& Sacco, B. 1985, in Data Analysis in Astronomy, ed. V. di Gesu, L. Scarsi, P. Crane, J. H. Friedman, \& S. Levialdi, 15

Bulgarelli, A., Gianotti, F., Trifoglio, M., et al. 2009, The Astronomer's Telegram, 2017, 1

Chaty, S., Zurita Heras, J. A., \& Bodaghee, A. 2010 [arXiv: 1012.2318]

Coe, M. J., Townsend, L. J., \& Udalski, A. 2010, The Astronomer's Telegram, 2597,1

Cusumano, G., La Parola, V., Romano, P., et al. 2010a, MNRAS, 406, L16

Cusumano, G., La Parola, V., Segreto, A., et al. 2010b, A\&A, 510, A48

D’Aì, A., Cusumano, G., La Parola, V., \& Segreto, A. 2010, The Astronomer's Telegram, 2596, 1

Dickey, J. M., \& Lockman, F. J. 1990, ARA\&A, 28, 215

Evans, P. A., Beardmore, A. P., Page, K. L., et al. 2009, MNRAS, 397, 1177

Gehrels, N., Chincarini, G., Giommi, P., et al. 2004, ApJ, 611, 1005

Guinan, E. F., Fitzpatrick, E. L., Dewarf, L. E., et al. 1998, ApJ, 509, L21

Habets, G. M. H. J., \& Heintze, J. R. W. 1981, A\&AS, 46, 193

Halpern, J. P. 2005, The Astronomer's Telegram, 572, 1

Kalberla, P. M. W., Burton, W. B., Hartmann, D., et al. 2005, A\&A, 440, 775

Kuulkers, E., Shaw, S., Paizis, A., et al. 2006, The Astronomer's Telegram, 874 1

Kuulkers, E., Shaw, S. E., Paizis, A., et al. 2007, A\&A, 466, 595

La Parola, V., Cusumano, G., Romano, P., et al. 2010a, MNRAS, 405, L66

La Parola, V., Cusumano, G., Segreto, A., et al. 2010b, The Astronomer's Telegram, 2594, 1

Landi, R., Masetti, N., Malizia, A., et al. 2008, The Astronomer's Telegram, 1539,1

Liu, Q. Z., van Paradijs, J., \& van den Heuvel, E. P. J. 2006, A\&A, 455, 1165

Masetti, N., Morelli, L., Palazzi, E., et al. 2006, A\&A, 459, 21

Sazonov, S., Churazov, E., Revnivtsev, M., Vikhlinin, A., \& Sunyaev, R. 2005, A\&A, 444, L37

Segreto, A., Cusumano, G., Ferrigno, C., et al. 2010, A\&A, 510, A47

Tomsick, J. A., Chaty, S., Rodriguez, J., Walter, R., \& Kaaret, P. 2009, ApJ, 701, 811

Ubertini, P., Lebrun, F., Di Cocco, G., et al. 2003, A\&A, 411, L131

Vercellone, S., D'Ammando, F., Striani, E., et al. 2009, The Astronomer's Telegram, 2019, 1

Walter, R., Zurita Heras, J., Bassani, L., et al. 2006, A\&A, 453, 133

Winkler, C., Courvoisier, T., Di Cocco, G., et al. 2003, A\&A, 411, L1 